\title{
Marc Bloch y eL \\ COMPROMISO DEL INTELECTUAL
}

\section{Carlos Antonio Aguirre Rojas ${ }^{1}$}

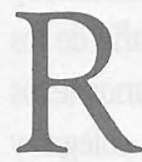

eferirse, en estos comienzos del tercer milenio cronológico, a la vida, a la biografía intelectual y a la obra del historiador francés Marc Bloch, es referirse, sin duda alguna, al itinerario personal e intelectual, y al trabajo del mas importante historiador que ha existido en todo el mundo occidental durante la primera mitad del siglo XX. Porque a medida que nos alejamos de la propia época y de las circunstancias generales en que ha vivido Marc Bloch, mas va ganando en influencia y en presencia cultural planetaria la específica contribución intelectual de este mismo historiador.

Y como toda obra y vida complejas y ricas, también la de Marc Bloch es una travesía que comprende múltiples dimensiones, que incluyen lo mismo su trabajo como historiador que su posicionamiento social y político frente a su propia circunstancia y su propio presente, junto a su labor como Profesor y formador de nuevas conciencias o su actividad práctica organizativa de difusión de un ambicioso proyecto de renovación historiográfica a través de la construcción de sus Annales d'Histoire Economique et Sociale, entre muchas otras. Múltiples dimensiones del 'ser en el mundo' de este importante personaje de los estudios históricos franceses y europeos de la primera mitad del siglo XX, que no casualmente siguen siendo, todavía hoy, objeto de reflexión y de debate entre los científicos sociales y los historiadores de todo el planeta.

Y así, lo mismo en Europa que en América Latina, en el Lejano Oriente que en Estados Unidos, en Rusia o en la India, vemos cada día multiplicarse las traducciones de sus principales textos lo mismo que los homenajes a su aporte intelectual y a su vida, dentro de una línea de tendencia que, en todas partes del mundo, avanza en el sentido de la revaloración creciente de sus concepciones teóricas sobre la historia y de sus principales descubrimientos e hipótesis de orden historiográfico, pero también de sus tomas de posición

1 Doutor em Economia pela Universidade Nacional Autônoma do México. Professor do Depto. de História da Unam. No Brasil, é autor dos livros: Tempo, duração e civilização. Percursos Braudelianos e Braudel, o mundo e o Brasil, ambos pela editora Cortez. 
frente a los conflictos políticos y a las excepcionales circunstancias históricas que han constituido su específico presente, su singular 'época' y su particular 'medio' o ambiente de existencia. A este respecto, resulta significativo el hecho de que en 1992 ha sido fundada en Paris una Association Marc Bloch que ha reunido a investigadores e historiadores de mas de 10 países del mundo y que ha publicado hasta hoy 5 entregas de la revista Cabiers Marc Bloch. En estos cinco números se encuentran, entre otras cosas, una abundante información acerca de las traducciones recientes y los homenajes en torno a la figura de Marc Bloch, artículos interesantes sobre su obra, su vida y el estado de los manuscritos y trabajos que componen su legado intelectual global, una primera Bibliografía de los principales artículos escritos desde 1944 sobre este mismo historiador, algunos texos inéditos del mismo Bloch, y una parte de su correspondencia con diversos colegas y alumnos. También podemos mencionar los trabajos de largo aliento que, recientemente, le han dedicado varios historiadores y científicos sociales ${ }^{2}$.

Entonces, lo mismo para recuperar sus sugerentes tesis contenidas en la hoy célebre Apología para la Historia y definir desde ellas lo que hoy debe ser una verdadera historia crítica, que para explicar de manera novedosa y compleja la historia agraria de algún país latinoamericano, pero también para rediscutir la función social del historiador y su compromiso con su propio presente y con sus circunstancias históricas, o para adentrarse una vez mas en el complicado tema de las claves de la singularidad de la historia de la civilización europea, los historiadores y científicos sociales de todo el mundo vuelven a mirar hacia la herencia intelectual de Marc Bloch, reproblematizando de nueva cuenta los elementos centrales de su legado cultural.

Por eso, sigue siendo útil y hasta necesario retornar a la lectura y al estudio sistemático de esos diferentes aspectos del universo blochiano, los que en la actualidad y todavía por un buen periodo de tiempo por venir, seguirán constituyendo lecciones importantes e imprescindibles para todos aquellos historiadores serios y científicos que, lejos de la historia oficial, positivista, acrítica y banal, quieran continuar desarrollando hoy la innovación historiográfica y el ejercicio de una historia realmente crítica.

2 Mastrogregori, M. El Manuscrito Intervumpido de Marc Bloch. México: FCE, 1998; y Introduzione a Bloch. Roma: Laterza, 2001. Dumoutin, 0. Marc Bloch. Paris: Presses de la Fondation Nationale des Sciences Politiques, 2000. Friedmann, S. W. Marc Bloch, sociology and geography. Cambridge: Cambridge University Press, 1996. FinK, C. Marc Bloch. A Life in History. Cambridge: Cambridge University Press, 1989. BLoch, E. Marc Bloch. Une biographie impossible. Limoges: Culture et Patrimoine en Limousin, 1997. AguiRRE RoJAs, C. A. Los Annales y la Historiografía francesa. México: Quinto Sol, 1996; y La Escuela de los Annales. Ayer, Hoy, Mañana. Villahermosa. UJAT, 2002. Igualmente vale la pena mencionar el libro de varios autores Marc Bloch aujourd'bui. Histoire comparée et sciences sociales. Paris: EHESS, 1990, y las Actas de un Coloquio celebrado en Estrasburgo en 1994, bajo el título Marc Bloch, l'bistorien et la cité. Strasbourg: PUS, 1997. 
Y también puede resultar instructivo y aleccionador, en esta época nuestra marcada por la existencia de ciertas tendencias posmodernas que promueven el desencanto general y la apatía y abstención absoluta de los científicos sociales y de los historiadores respecto de su propio compromiso social, volver a examinar la actitud específica que ha tenido Marc Bloch respecto de su propio presente, asumiendo de manera radical ese compromiso que el historiador, y mas en general todo intelectual u hombre de cultura, debe de tener frente a su sociedad y frente al mundo en el que vive, en virtud de que es en estos últimos dentro de los cuales ejerce y despliega los resultados esenciales de su propia actividad intelectual.

Tratando entonces de señalar de manera general, algunos de esos aportes principales contenidos en la obra de Marc Bloch, y también algunas de esas lecciones de su toma de posición respecto de los problemas sociales y las condiciones históricas en que él ha vivido, puede ser útil tratar de reconstruir las líneas esenciales y las etapas fundamentales del entero periplo intelectual que ha recorrido este gran historiador, autor tanto del bello libro sobre La Sociedad Feudal, como de la hoy ampliamente difundida Apología para la Historia u Oficio de Historiador ${ }^{3}$, pero también de las agudas reflexiones contenidas en su libro sobre La Extraña Derrota, o en sus artículos escritos al final de su vida y publicados en los Cabiers Politiques, igual que en sus Cuadernos de notas personales titulados por él mismo como 'MEA'.

Concentrándonos de este modo en estas dos dimensiones de la compleja biografía personal e intelectual de Marc Bloch, que son las contribuciones principales de su obra y las enseñanzas de su actitud frente al presente, quizá podamos continuar avanzando en este necesario camino de la urgente recuperación de su legado general tanto a los estudios históricos como a la cultura de las ciencias sociales de nuestra propia y mas candente actualidad.

3 Es bien sabido que de este importante libro, que Marc Bloch dejó inconcluso por razones de una clara elección de tipo político, existe ahora una nueva edición, que integra tanto la primera redacción del texto como la redacción 'definitiva', junto a ciertas hojas de esbozo de ambas redacciones, permitiéndonos tener ahora una idea mucho mas rica y mas aproximada del esquema completo y del verdadero carácter de la reflexiones que Bloch deseaba concretar allí. Cf. esta edición Apología para la Historia u Oficio de Historiador. México: FCE, 1996. Aunque esta edición y su primera reimpresión en 1998, se han agotado muy rápido, este libro no ha vuelto a ser reeditado después, por razones extrañamente inexplicables.

4 Curiosamente, no existe hasta hoy traducción al español de este importante libro de Marc Bloch sobre la derrota de Francia en la segunda guerra mundial, libro en el cual nuestro historiador establece una radiografía crítica implacable de la sociedad francesa en vísperas de ese mismo conflicto bélico iniciado en 1939. Cf. L'etrange defaite. Paris: Gallimard, 1990, que incluye también algunos de los artículos publicados en los Cahiers Politiques mencionados. Sobre el cuaderno de notas MEA, cf. MAsTrogregori, M. 'Due Carnets inediti di Marc Bloch (1917 - 1943): "Quelques notes de lecture" et "Mea"', en la Rivista Storica Italiana, vol. CX, 3, Roma, 1998. 
Con lo cual podremos 'mirar' el entero periplo intelectual de Bloch desde una doble óptica general. Tanto en su dimensión como hombre de cultura que se posiciona frente a los dramáticos hechos que conforman a la historia francesa y europea de los últimos años del siglo XIX y de los años del 'primer siglo XX' que corre desde 1914 hasta 1945, como también en el nivel del historiador y científico social que, mediante múltiples intervenciones historiográficas, llega a ser capaz de gestar, junto a Lucien Febvre, una verdadera 'revolución en la teoría de la historia' dentro de los estudios históricos entonces dominantes en Francia, en Europa y en la mayoría del mundo occidental.

Porque cuando observamos en su conjunto el entero itinerario de Bloch, resulta evidente que él no ha sido nunca un intelectual de una izquierda militante, siendo lo mas probable que no ha llegado incluso nunca a ser miembro regular de algún partido político. Lo que entonces, excluye claramente en su caso la figura, común en otro tipo de personajes similares, del intelectual activamente vinculado a los movimientos políticos y prácticos de su propia época. Pero, si este no es el caso de Marc Bloch, si es claro en cambio que, a lo largo de toda su vida, Bloch ha sido un intelectual que reflexionaba seria y profundamente sobre los sucesos políticos y sobre las circunstancias sociales en las cuales vivía cotidianamente, siendo un pensador que, de manera radical y sin concesiones, era absolutamente honesto consigo mismo y con los otros, asumiendo totalmente el compromiso con su trabajo, con su sociedad y con su propio oficio. Es decir, un intelectual atento a los problemas de su época, preocupado por los acontecimientos y situaciones que vivía, y que bajo todas las formas posibles combatía de manera implacable la mentira, el disimulo y la componenda, sin importar donde se originaran estos.

Un intelectual que era hasta tal punto fiel a sus principios, a sus puntos de vista y a su específica evaluación de las situaciones en que a lo largo de su vida desplegó su trabajo y su actividad en general, que era capaz de ser consecuente con esos principios y evaluaciones incluso al punto de poner en riesgo su propia vida, y hasta el punto del sacrificio personal, el que lamentablemente se concretó precisamente en el año de 1944. Historiador agudo y crítico, y ciudadano realmente comprometido con su país y con su sociedad, que a través de un arduo trabajo de reflexión crítica fue poco a poco radicalizando sus posiciones políticas y asumiendo mas integralmente su compromiso social, para terminar sacrificando, primero su obra de historiador y luego su propia vida, a esa conciencia aguda de su ineludible deber como miembro de la sociedad francesa y europea, ubicado dentro de los movimientos de la resistencia social a la amenaza terrible de la barbarie nazi entonces viva y actuante.

Por otro lado, y en la segunda dimensión de la vida de Marc Bloch que hemos apuntado, es también claro que nuestro autor se afirma como el mas importante 
historiador francés, europeo y del mundo occidental de la primera mitad del siglo XX, transformando mediante sus escritos y su obra académico práctica los cánones entonces vigentes de lo que debía ser la practica cotidiana del oficio de historiador. Y ello, no solo a través de la ya mencionada revolución teórica de los estudios históricos franceses y europeos, concretada en el importante proyecto de lo que mas adelante será erróneamente llamado la 'Escuela de los Annales's, sino también por la vía de la apertura constante de nuevos campos problemáticos, de la invención heurística de nuevos modelos teóricos y nuevos conceptos y teorías historiográficas, del descubrimiento y explicitación de nuevos paradigmas metodológicos, y del desarrollo de nuevas aportaciones historiográficas en los temas concretos que el mismo ha ido abordando a lo largo de su propia carrera de historiador.

Obra polifacética y compleja que lejos de ser la obra de un 'especialista' en temas 'medievales', es mas bien una obra siempre atenta a los problemas 'de orden general' que preocupan a todo historiador, desde su vasta y acertada definición de 'ciencia de la obra de los hombres en el tiempo', preocupada en esclarecer el enorme tema de los mecanismos y las modalidades del 'cambio histórico en la historia', y siempre orientada hacia la reflexión explicita de la función y de los impactos sociales del propio trabajo del historiador, según las propias ideas y concepciones del mismo Marc Bloch.

Veamos entonces desde este doble emplazamiento analítico las etapas principales de ese rico itinerario social e intelectual del autor de Los Reyes Taumaturgos y de los Caracteres originales de la bistoria rural francesa.

\footnotetext{
Sobre este punto, que nos sea permitido referir al lector a nuestros trabajos antes citados Los Annales y la bistoriografía francesa y también La Escuela de los Annales. Ayer; boy, mañana. Tambien nuestros libros Itinerarios de la bistoriografia del siglo XX. La Habana: Juan Marinelo, 1999; Breves Ensayos Críticos. Morelia: Universidad Michoacana, 2000; América Latina. Historia y Presente. Morelia: Jitanjáfora, 2001; y Antimanual del mal historiador. México: La Vasija, 2002. Para otras explicaciones sobre la historia de esta mal llamada 'Escuela de los Annales' y sobre el rol de Marc Bloch en ella, cf. Dase, F. La bistoria en migajas. Valencia: Alfons el Magnanim, 1988. BuRKE, P. La revolucion historiográfica francesa. La escuela de los Annales: 1929 - 1989. Barcelona: Gedisa, 1993. ReIs, J.C. Nouvelle Histoire e Tempo Histórico. Sao Paulo: Ática, 1994. Mastrogregori, M. Il genio dello storico. Le considerazione sulla storia di Marc Bloch e Lucien Febure la tradizione metodologica francese. Roma: Edizione Scientifiche Italiane, 1987. También es útil ver la compilación organizada por MiddeiL, M. y SammiER, S. Alles Gewordene bat Geschichte. Die Schule der Annales in ibren Texten. Leipzig: Reclam Leipzig, 1994.
} 


\section{LA ETAPA Formativa IniCial (1886 - 1908)}

Los primeros veintidós años de Marc Bloch son los años de su formación intelectual originaria, en los cuales van a definirse tanto los rasgos generales de su personalidad individual y de su perfil intelectual, como su vocación clara en torno del ejercicio de la historia. Bloch nace en Lyon el 6 de julio de 1886, en el seno de una familia modestamente acomodada que pocos años después se trasladará a Paris, en donde habrá de transcurrir el resto de su infancia y de su adolescencia y hasta el inicio de su primera juventud. Y será el propio Bloch el que afirmará que tuvo una infancia feliz, rodeado del cariño de sus padres, lo que redundará en un carácter bastante seguro de si mismo y en una personalidad fuerte, que era capaz de sostener tenazmente sus principales proyectos, y que poseía el temple necesario para poder hacer frente a las adversidades y catástrofes de las distintas situaciones difíciles en que Bloch se verá involucrado varias veces durante su vida.

Era hijo de Gustave Bloch, un conocido historiador especialista de la antigüedad romana, que llegará a ser titular de una Cátedra en la Sorbonne. Y es importante esta filiación familiar de Marc Bloch, que le dará acceso, desde muy pequeño, a los tesoros importantes de la bien provista Biblioteca de su padre, y a los contactos con importantes representantes de ese medio de la historiografía francesa en que este último trabajaba. Con lo cual y desde esta misma etapa formativa inicial, Bloch conocerá los principales debates historiográficos de su época, leyendo las principales revistas de historia de aquellos tiempos y conociendo incluso personalmente, en su propia casa, a varios de los protagonistas centrales de esos mismos debates referidos. Al mismo tiempo, y junto a estas fuentes directas del conocimiento de la historia que entonces comienza a aprender, nuestro autor se convertirá sin problemas en el propio alumno de su padre, a quien el agradece los primeros elementos de su 'formación como historiador' en el Prefacio de su primer libro importante de historia que es Los Reyes Taumaturgos.

Recibiendo entonces esta impronta paterna que él mismo califica de imborrable, y que lo orientará claramente hacia los estudios históricos, Bloch tendrá también, en su condición de hijo de Gustave Bloch, algunas puertas abiertas dentro los círculos diversos de la elite de los historiadores franceses de finales del siglo XIX y principios del siglo XX, lo que le permitirá en varios momentos un acceso mas ágil a los medios académicos y universitarios dentro de los cuales desarrollará sus principales empresas culturales.

Aunque de una manera para nada lineal, sino mas bien cambiante y contradictoria. Porque Bloch pertenece también a una familia de origen judío, que aunque no lo era de religión, y aunque se hallaba totalmente asimilada a la vida y a la 
historia de la sociedad francesa, no por ello dejará de tener consecuencias importantes dentro de su propio itinerario personal e intelectual. Pues es bien sabido que Marc Bloch será víctima, en varias ocasiones, del antisemitismo reinante dentro de Francia aún en su propia época, antisemitismo que le impedirá llegar a ser Profesor en el prestigiado Collège de France y también Director de la Ecole Normale Superieure en la que el mismo había estudiado años atrás. Y será también su condición judía la que le implicará ser desposeído de su Cátedra en la Sorbonne, durante la segunda guerra mundial, provocando igualmente el allanamiento de su departamento parisino por parte de los nazis, así como parte de sus desplazamientos y fallidos proyectos de emigrar a Estados Unidos en esos mismos años de 1939 - 1944. Pero también, y en virtud de ese mismo origen y condición judíos, es que Marc Bloch va a poseer y a reproducir ese cosmopolitismo extraordinario y esa amplitud de horizontes que caracterizan a la gran mayoría de los pensadores judíos europeos de los últimos 150 años. Porque desde Marx y hasta Carlo Ginzburg o Immanuel Wallerstein, y pasando por Emile Durkheim, Sigmund Freud, la Escuela de Frankfurt, Norbert Elias o Walter Benjamin, es clara la enorme y profunda contribución que esta tradición de pensadores judíos ha hecho a la cultura de las ciencias sociales contemporáneas ${ }^{6}$.

Por ello, Bloch habla y/o lee sin ninguna dificultad, junto al francés, el alemán, el inglés y el italiano, además de tener sólidos conocimientos de griego y de latín, y de comprender aceptablemente el español. Y entonces, y gracias a este poliglotismo común a muchos de esos pensadores judíos mencionados, nuestro historiador estará enterado tanto de los trabajos principales producidos en toda Europa y en Estados Unidos respecto de sus distintos temas abordados, como también de los principales debates historiográficos nacionales de prácticamente todo el espacio de Europa Occidental. Algo que se hará evidente, por ejemplo, en la variedad de fuentes y en la amplitud de casos comparados y examinados en su importante obra sobre La Sociedad Feudal.

Gozando así de esta amplitud de horizontes culturales y de esta excepcional formación histórica derivada de su núcleo familiar, Bloch va a estudiar y a aprobar sin dificultad, e incluso con premios y primeros honores, sus estudios realizados en el prestigioso Liceo Louis Le-Grand de Paris, y luego en la Escuela Normal Superior, la misma en la que estudiarán mas tarde o mas temprano Lucien Febvre, Raymon Aron, Jean-Paul Sartre 0

\footnotetext{
6 Sobre esta importante contribución de los pensadores de esta tradición judía cf. Echeverrí, B. "Benjamín: Mesianismo y Utopía” en el libro Valor de uso y Utopía. México: Siglo XXI, 1998. NeTTeL, P. "Marc Bloch: un historiador entre la civilización y la barbarie" y Aguirre Rojas, C.A. "Norbert Elías, historiador y crítico de la modernidad" ambos en el libro Aproximaciones a la modernidad. México: UAM Xochimilco, 1997, y AguiRre Rojas. "Walter Benjamín y las lecciones de una historia vista a 'contrapelo"' en revista Secuencia, 52, México, 2002.
} 
Michel Foucault, entre tantos otros importantes intelectuales franceses del siglo XX. Estudios que, muy fácilmente, se verán coronados con la precoz obtención de su Diploma de Estudios Superiores en 1907 y de su Diploma de Agregación en 1908.

Cursando sus estudios de Historia y Geografía en dichaEcole Normale Superieure, Bloch va a aprender allí los distintos elementos de la tradicional y puntillosa historia erudita y positivista entonces reinante en los estudios históricos franceses, asimilando las lecciones de la lectura y el examen sistemático de los textos y la crítica interna y externa de las fuentes. Una historia metódica y acartonada con la que él mismo romperá radicalmente mas adelante, cuando funde en 1929, junto a Lucien Febvre, los Annales de Historia Económica y Social, y a la que ya en esas mismas épocas comienza a problematizar y a cuestionar, como lo atestigua su Cuaderno de notas escrito en 1906 y titulado Metodología histórica, y que felizmente se ha conservado hasta nuestros días?.

En estas breves notas, Bloch afirma sin ambages que la historia que existe entonces no es una ciencia, criticando frontalmente su carácter puramente descriptivo y acumulativo, y defendiendo vigorosamentelanecesidaddeincorporary construirdentrodelosestudios históricos un verdadero 'método analítico', lo mismo que el establecimiento de claras y reales 'problemáticas' o temas pertinentes de estudio, yel necesario 'análisis de los acontecimientos' para constituirlos en verdaderos fenómenos dignos de estudio. Observaciones críticamente agudas frente a la historia positivista entonces dominante en Francia, que revelan claramente el hecho de que Marc Bloch no solo se ha formado abrevando en las obras de los mismos historiadores, sino también en la lectura y reflexión de, por ejemplo, los trabajos de la escuela sociológica de Durkheimy de su revista L'Annee Sociologique, o en las obras de los filósofos de aquella época, lo mismo que en una muy sólida asimilación de las lecciones de la escuela francesa de geografía de Vidal de la Blache ${ }^{8}$ en algunos textos de la economía política entonces difundida dentro del hexágono francés.

Porque es importante insistir en el hecho de que Bloch va a formarse inicialmente en un periodo en el cual la explosión de las ciencias sociales va a afirmarse con cierta fuerza dentro de la Francia de aquellos tiempos. Yentonces, al mismo tiempo que aprende esa historiapuramenteerudita, nuestro historiador en ciernes se convierte en un lector muy atento de la economía, de la filosofía, de la geografía y de esa nueva Sociología durkheimiana, cuya impronta reconocerá claramente en su célebre Apología para la Historia.

Este breve texto, Metodología Histórica, está incluido en el libro de Marc Bloch, Historia e Historiadores. Madrid: Akal, 1999.

8 En torno de estas presencias de la sociología durkheimiana y de la geografia vidaliana en la obra de Bloch, cf. el libro de FrIEDMn, S. Marc Bloch, Sociology and Geography. Encountering changig disciplines, antes citado. 
E igualmente, y desde esa misma etapa formativa inicial, Bloch va a estar muy al corriente de la revista fundada por Henri Berr bajo el título de Revue de Synthèse Historique, en la que incluso va a publicar su primera reseña crítica de un libro y algunos de sus primeros artículos, en los años de 1911 y en adelante? ${ }^{9}$ Publicación que atestigua acerca de una natural 'afinidad electiva' entre este proyecto innovador de Henri Berr, también encaminado a desmontar y a superar a esa viejay limitada historia positivista francesa, y el itinerario blochiano que camina, ya desde aquellos tempranos tiempos, en esa misma dirección intelectual.

Un último elemento importante a señalar en esta etapa inicial es el del hecho de que Bloch va a autodefinirse como miembro de esa generación de jóvenes franceses que vivieron de cerca y acompañaron directamente al muy resonado Affaire Dreyfus, que dividió prácticamente a Francia en dos claros campos opuestos, y que le permitió a Marc Bloch no solo cobrar conciencia clara del antisemitismo todavía imperante dentro de su propio país, sino que reafirmó también su clara toma de posición progresista y liberal, a la que no habrá de renunciar nunca y a la que, por el contrario, irá radicalizando progresivamente hacia la izquierda conforme madure y avance en su propio trayecto intelectual.

\section{Los Primeros Trabajos como Historiador (1909 -1922)}

El segundo periodo de la vida de Marc Bloch comienza con la doble estancia académica que va a realizar, en el año académico de 1909, en dos importantes Universidades alemanas de aquellos tiempos, que son la Universidad de Berlín y la Universidad de Leipzig. Dos Universidades que concentran a parte de los historiadores mas de vanguardia que entonces existen dentro de la historiografía alemana, y en las cuales Bloch va a conocer mas a fondo y a asimilar creativamente los principales aportes de esa misma historiografía germanoparlante, que es entonces dominante dentro del conjunto de los estudios históricos europeos y occidentales.

Porque no hay que olvidar que durante los años de 1870 a 1914, y antes de los terribles golpes sufridos por las culturas alemana y austriaca a consecuencia de la primera y la segunda guerra mundiales, pero sobre todo del nazismo, esa misma cultura de habla germana fue en verdad la cultura mas desarrollada, innovadora y realmente dominante

9 Sobre estas primeras publicaciones de Marc Bloch, cfr, la 'Bibliographie' incluida en el tomo 2 del libro Melanges Historiques. Paris: Serge Fleury/EHESS, 1983. 
dentro del conjunto del espacio europeo e incluso occidental. Lo que entonces, constituyó al llamado 'viaje a Alemania' en una actividad o estancia prácticamente obligada dentro del proceso formativo general de todo historiador serio, que pretendiese estar a la altura de los mas importantes desarrollos de la disciplina en aquellos años. De este modo, y siguiendo entonces esta misma práctica, habitual dentro de la propia corporación de historiadores de aquellas épocas, Bloch va a realizar dicha estancia de estudios en Alemania, adquiriendo así todos los principales elementos de esta cultura historiográfica de avanzada de acuñación germana. Y es claro que muchos de los perfiles específicos de la obra de Marc Bloch serían incomprensibles sin la consideración de esta matriz germana de su formación como historiador, lo que por ejemplo se hace particularmente evidente en los temas y en el argumento particular de su libro sobre la Historia Rural Francesa ${ }^{10}$.

Matriz germana del pensamiento blochiano, que en primer lugar alude al propio aprendizaje de la centralidad y de los contenidos esenciales de las ramas específicas de la bistoria económica y de la bistoria social. Dos ramas que, mientras que en Francia eran totalmente marginales o hasta inexistentes, eran en cambio campos muy desarrollados y debatidos dentro de la Alemania en la que va estudiar y a continuar su formación el mismo Bloch. Entonces, y gracias a este 'viaje a Alemania', nuestro autor va a entrar en contacto con la importante revista Vierteljahrschrift fur Sozial und Wirtschaftsgeschichte, que en esos años de inicios del siglo XX, es sin duda la mas importante revista de historia económica de toda Europa. Y también, va a tomar cursos y a entrar en contacto directo con la obra de autores como Karl Lamprecht, impulsor de la Kultur Gescbichte, o como Karl Bücher, Georg von Below, Gustav Schmoller o R. Kötzschke, que en aquellos tiempos están renovando e inventando las nuevas formas de la historia económica, de la geografía histórica, de la antropogeografía y de la historia del paisaje, de la tierra, o de la ocupación del territorio, entre otros.

De modo que todos los interesantes ensayos que conocemos de Marc Bloch, publicados en los años treintas dentro del campo de la moderna y entonces pionera historia agraria o rural, y relativos a la configuración y evolución históricas de las formas del paisaje, las formas del poblamiento rural, las formas del hábitat, y los estudios y encuestas sobre los planes parcelarios, etc., son todos ensayos que tienen una deuda profunda con las lecciones aprendidas por el autor de la Historia Rural Francesa durante este año académico de 1909 realizado en Alemania. Y no es por casualidad que,

${ }^{10}$ Pierre Toubert ha desarrollado muy convincentemente esta clara influencia alemana en dicha obra de Marc Bloch, en su Prefacio a los Caracteres Originaux de l'Histoire Rural Francesa, traducido al español y publicado en la revista Argumentos, 26, México, 1997, antes citada. 
a lo largo de toda su vida, Bloch se mantendrá muy atento al conjunto de los nuevos desarrollos producidos por esta historiografía alemana, la que revisará sistemáticamente, y sobre la que redactará, por ejemplo, varios Boletines críticos publicados en la Revue Historique a partir de 1928.

Y también, es gracias a esta estancia alemana que Bloch entrará en contacto con la obra de Henri Pirenne, obra que como es bien sabido, tendrá una influencia decisiva y central en todo el trabajo y en toda la actividad intelectual de nuestro autor $^{11}$. Porque de Pirenne, Bloch no solo va a recoger la importante lección de las implicaciones y vigencia del método comparativo dentro de la historia, sino también y mas ampliamente, el propio modelo de una historia interpretativa y crítica, atenta a la centralidad de los hechos económicos dentro de la historia, y siempre orientada mas allá de los estrechos marcos nacionales o locales. Es decir de un tipo de historia que será en mucho el que habrán de impulsar y promover los 'primeros Annales', durante el período de los años de 1929 a 1941.

Entre 1909 y 1912 Bloch va a obtener una beca de la Fondation Thiers que le permitirá dedicarse durante tres años completos solo a la investigación, lo que habrá de fructificar en la escritura de los primeros trabajos publicados, en los que lentamente comenzarán a delinearse algunas de las líneas fundamentales de lo que, años después será su Tesis de doctorado, junto también a algunas de sus investigaciones posteriores. Y en especial, será en 1913 que vea la luz el primer trabajo de mas grande aliento de Marc Bloch, es decir su monografía de historia regional titulada L'lle de France (Les pays autour de Paris), publicada dentro de la colección Les Régions de la France, impulsada una vez más por el grupo de Henri Berr y de la Revue de Synthèse Historique.

Verdadero modelo de lo que debe ser una historia regional cientificamente concebida, este primer texto largo de Marc Bloch va a definir claramente los limites reiterados que padecen la inmensa mayoría de los estudios de historia local o de historia regional tradicionales. Porque afrontando directamente la pregunta crucial que debe asumir todo historiador local o regional, y que es la de ¿a quien le interesa este trabajo sobre la historia local del pequeño pueblo dex, o sobre la historia regional de la región y?, nuestro autor va a responder que, para escapar a la obligada respuesta de que dicho trabajo solo interesará a los habitantes de ese mismo pueblo x o esa región y, el historiador

\footnotetext{
${ }^{11}$ Sobre esta crucial relación entre Bloch y Pirenne, cf. la Correspondencia publicada por Bryce y Mary Lyon, The birth of Annales bistory: the letters of Lucien Febre and marc Bloch to Henri Pirenne (1921 - 1935). Bruxelas: Commission Royale d'Histoire, 1991. Sobre la influencia de la obra de Pirenne dentro del proyecto de los 'primeros Annales' cf. Aguirre Rojas, C.A. La escuela de los Annales. Ayer, Hoy, Mañana, antes citado.
} 
está obligado a conectar, de manera explicita y creativa, los elementos de la historia local o regional que aborda con los elementos esenciales de la bistoria general. Es decir que la única definición posible de una historia local o regional científicamente concebida, es aquella que aborda 'una pregunta de interés general planteada a los documentos que provee una región particular ${ }^{\prime 2}$. Porque solo al precio de abordar estos problemas generales, que le preocupan y ocupan a todos los historiadores, es que será posible construir una historia regional o local no limitada a ser una simple monografía descriptiva de alcances pequeños y de igualmente acotado interés.

Mas adelante, y al concluir esta beca de la Fundación Thiers, Bloch será Profesor, primero en el Liceo de Montpellier en el año académico de 1912 - 13, y luego en el Liceo de Amiens, durante el año académico de 1913 - 1914. Y es en este último lugar en donde va a sorprenderlo el estallido de la primera guerra mundial, que provocará su movilización hacia el frente a principios denau agosto de 1914, y con ella la dura experiencia de una primera guerra vivida y protagonizada por el propio Bloch. Una guerra que, a la vez que aguza su posición republicana y su conciencia de la crisis que vive la civilización europea, va también a permitirle mirar ciertos fenómenos creados por esa misma situación excepcional del frente de guerra con la mirada atenta y escrutadora del historiador realmente crítico.

Porque en 1921, y como uno de los resultados importantes de su experiencia vivida en las trincheras, Marc Bloch va a publicar su sugestivo ensayo titulado "Reflexiones de un historiador acerca de los bulos surgidos durante la guerra"13. En este último, y considerando a la guerra como una suerte de inmenso 'experimento de psicología social', nuestro autor va a preguntarse cómo es que se originan los diversos rumores surgidos y difundidos durante esa primera guerra mundial, y mediante qué mecanismos se afirman

12 Para esta definición cf. L'lle de France (Le pays autour de Paris), en el libro Mélanges Historiques, tomo 2 , p. 786, antes citado. Curiosamente, este primer trabajo extenso de Marc Bloch no ha sido traducido, hasta donde sabemos, a ningún idioma mas allá de la versión original francesa. Esta preocupación de Marc Bloch en torno de las condiciones necesarias para que la historia local o regional no fuese una empresa de sabios locales y de historiadores amateurs, promovida por intereses extrahistóricos y limitada en sus resultados a monografías intrascendentes, carentes de interés y puramente anecdóticas, reaparece en varias de su reseñas críticas y de sus ensayos breves publicados en los Annales d'Histoire Economique et Sociale, algunos de los cuales han sido recientemente reeditados en el libro La terre et le paysan. Agriculture et vie rurale aux XVIIe et XVIIIe siecles. Paris: Armand Colin, 1999.

13 Sobre esta experiencia personal de Bloch dentro de esa primera guerra vale la pena consultar sus textos reunidos en el libro Ecrits de guerre 1914 - 1918. Paris: Armand Colin, 1997. El artículo mencionado sobre los bulos o rumores surgidos durante la guerra está incluido en el libro Historia e Historiadores, antes citado. Otro ejemplo muy interesante de estos fenómenos de la conciencia colectiva, puede verse en el libro de LEFEBVRE, G. El gran pánico de 1789. Barcelona: Paidos, 1986. 
y propagan en la conciencia colectiva, pero sobre todo, cuál es el fundamento que determina y discrimina aquellos rumores que tienen éxito y que prosperan, frente a aquellos otros que mueren o desaparecen sin trascender el limitado ámbito del simple error o confusión individual.

Entonces, y recordándonos que ha sido esa situación excepcional de la guerra, en donde los periódicos no circulan normalmente y en donde la credibilidad de las noticias 'oficiales' cae por lo suelos, la que nos devuelve a una circunstancia similar a la del medioevo o a la de la antigüedad, en la que la tradición oral es el medio de comunicación por excelencia, y en la que los rumores o bulos se encuentran entonces a la orden del día. Con lo cual, pueden prosperar y divulgarse ampliamente aquellos rumores que, nacidos de una simple apreciación falsa o parcial de algún hecho o fenómeno, terminan por corresponderse con los miedos, los prejuicios, las fantasías sociales o los deseos y emociones fuertes previamente existentes dentro de esa misma conciencia colectiva.

Porque apoyándose en las investigaciones mas recientes ofrecidas por la psicología del testimonio, que hacen evidente la fragilidad y limitación de cualquier declaración o descripción de todo tipo de testigos, Bloch demuestra que el error, la imprecisión y la mentira están presentes en prácticamente la totalidad de los documentos con los que trabaja un historiador. Pero el paso desde el simple error de un testigo o de un testimonio, hasta la conformación de ese fenómeno de psicología social que es la difusión masiva y amplia de un verdadero rumor social, solo se franquea si dicho error individual es capaz de reflejar de manera adecuada esas estructuras preexistentes de la conciencia colectiva, que son la base de la formación de todo tipo de mitos, leyendas populares y creencias sociales colectivas, y en consecuencia, también de la formación y propagación de esos rumores o falsas noticias.

Habiendo obtenido en 1920 su título de Doctor, con un trabajo sobre el tema Rois et serfs. Un chapitre d'bistoire capétienne ${ }^{14}$, Marc Bloch comenzará desde 1919 a trabajar en la Universidad de Estrasburgo, en donde construirá su alianza intelectual con Lucien Febvre, y en donde, durante mas de tres lustros, habrá de continuar sus actividades académicas, y mas en general su diverso y rico periplo intelectual.

\footnotetext{
${ }^{14}$ Este trabajo de tesis doctoral de Marc Bloch permaneció sin ser reeditado durante mucho tiempo. Recientemente ha sido vuelto a publicar bajo el título Rois et serfs et autres écrits sur le servage. Paris: La Boutique de l'Histoire, 1996.
} 


\section{En el Camino hacia la Gran Historia (1923-1931)}

La tercera etapa del itinerario blochiano es una etapa en la que se combinan, de un lado la concreción de sus primeros trabajos fundamentales dentro de la historiografía francesa y europea que le es contemporánea, y del otro la clara maduración lenta de varios proyectos intelectuales que solo habrán de consolidarse y de culminarse en el siguiente momento de su trayecto biográfico intelectual.

Así ya a principios de 1924, aparece publicado el libro de Los Reyes Taumaturgos, un libro que a la vez que prolonga y ahonda algunas de las preocupaciones ya esbozadas en el artículo antes mencionado sobre los rumores o falsas noticias de la guerra, constituye también la mas clara propuesta de Marc Bloch para el abordaje histórico crítico de los temas de la historia cultural. Porque tomando como problema central, la explicación de la curva global de evolución de la creencia colectiva popular en el poder taumatúrgico de los reyes de Francia y de Inglaterra, desplegado entre los siglos XIII y XVIII aproximadamente, lo que en verdad va a ofrecernos Bloch en este interesante libro es todo un modelo general de análisis para el estudio de la bistoria de las creencias populares colectivas.

Resituando entonces esa explicación de la creencia en el poder curativo de los monarcas franceses e ingleses, durante esos siglos del final del feudalismo y de principios del capitalismo, en función de los diversos niveles y realidades que le dan sentido y coherencia, Bloch va a construir un modelo que propone explicar los fenómenos culturales a partir de un registro múltiple de las varias dimensiones que en ellos se condensan, y que en su específica superposición, permiten comprender su compleja trama y evolución particulares.

Lo que, para el caso abordado en este libro, nos remite tanto al uso conciente y coyuntural que hacen los monarcas débiles, o en otro caso, urgidos de popularidad y de consenso, de ese mismo 'milagro' taumatúrgico, como, en una segunda dimensión, al claro conflicto cíclico y recurrente entre el poder secular de los reyes y el poder real y profano de la Iglesia. Porque en la eterna querella sobre el predominio de los reyes o el de los Papas, y en la disputa económica y social descarnada en contra de la Iglesia y de su para nada etérea presencia terrenal en este mundo, en el momento del tránsito del mundo feudal al mundo moderno, y de la inevitable decadencia del poder general de la Iglesia, se encuentra también una de las explicaciones importantes de la dinámica y de la afirmación variable de esta misma creencia popular colectiva.

Pero, y todavía en una tercera dimensión cultural mas profunda, esta creencia 
de las clases populares en el milagro del 'toque real' que cura las escrófulas, hunde sus raíces en la milenaria y ancestral concepción de los pueblos que conciben a sus gobernantes y dirigentes, sean sacerdotes, brujos, reyes 0 jefes militares, como seres 'sobrenaturales' y extraordinarios, dotados de poderes especiales y únicos, y rodeados de circunstancias siempre excepcionales y maravillosas. Creencia en la condición de excepcionalidad de todos aquellos hombres que llegan a ocupar algún puesto de poder - político, religioso, militar, económico o social de cualquier género -, que no hace mas que refrendar la idea, ampliamente arraigada durante la larga historia humana precapitalista, de que el mundo en su conjunto posee una doble significación, y una doble existencia, tanto profana, material y hasta vulgar, como también otra dimensión 'mágica', 'sobrenatural', 'profunda e inmaterial'. Concepción de esa condición 'no ordinaria' de los dirigentes y monarcas, que a pesar de la 'desacralización del mundo' impulsada por la modernidad capitalista, y del iluminismo racionalista también moderno, parece sin embargo persistir tenazmente hasta hoy en torno de los presidentes y los personajes de la alta política de todas las naciones del orbe.

De este modo, y desmontando magistralmente los varios niveles complejos imbricados en este fenómeno cultural de la creencia estudiada, Marc Bloch nos entrega un modelo de historia cultural que no es para nada 'historia de la mentalidades', y que en la actualidad solo parece haber sido recuperado y prolongado por el historiador italiano Carlo Ginzburg ${ }^{15}$. Y también, y escribiendo con ello una obra realmente pionera de la moderna antropología histórica, este libro de Los Reyes Taumaturgos que aquí comentamos, le permite a Bloch desentrañar el mecanismo general de la manera en que el Estado o el poder político se afirma a través de ciertas estructuras culturales vinculadas a esas creencias populares, a las que recupera, instrumentaliza y utiliza de una manera velada pero muy conciente ${ }^{16}$.

Y al mismo tiempo que elabora esta original obra de historia cultural, Bloch comienza a proyectar, junto con Lucien Febvre, la fundación de una nueva revista

\footnotetext{
15 Lejos de la amorfa y casi siempre intrascendente historia de las mentalidades de la tercera generación de los Annales, el modelo de Bloch es claramente perceptible, en cambio, en el interesante libro de Carlo Ginzburg Historia Nocturna. Barcelona: Muchnik, 1991. El libro de Marc Bloch, Los Reyes Taumaturgos, esta editado en México por el FCE, 1988.

16 Se encuentra entonces también en este libro la explicación del modo mismo en que se construye una determinada dominación política, pero también una dominación cultural o ideológica concomitante y complementaria, desde la manipulación y el uso sesgado que los poderes políticos dominantes hacen de las corrientes profundas de esa conciencia colectiva de los pueblos. Sobre este punto cf. el artículo de Ulrich Raulf, "República y carisma. Marc Bloch y el prodigio moderno" en la revista Argumentos, num. 26, México, antes citada.
} 
internacional de historia económica, proyecto que habiendo sido concebido desde el mismo final de la primera guerra mundial, solo alcanzará a concretarse, modificado, hasta 1929, con la fundación de los hoy célebres Annales d'Histoire Economique et Sociale. Un proyecto que, en el momento de su concepción original, en 1921, estaba muy concientemente orientado a sustituir la entonces golpeada y declinante hegemonía historiográfica alemana, por una nueva hegemonía historiográfica, inicialmente concebida como un proyecto de los 'aliados', pero que finalmente terminará radicándose solo dentro del espacio cultural del hexágono francés. Es también en este periodo, que Bloch ha comenzado a trabajar en el proyecto de la que será su mas importante obra de historia, en los dos volúmenes del libro de La Sociedad Feudal. Un libro que va a consumir lo mejor de su labor intelectual de quince años, y en el que se condensan las profundas, agudas y vastas lecturas de Marc Bloch en torno a la historia de la civilización europea durante el periodo de su infancia feudal o medieval ${ }^{17}$.

Al mismo tiempo que intenta echar a andar esa nueva revista internacional de historia económica, y que trabaja en las primeras etapas de su libro sobre la sociedad feudal, nuestro autor comienza a ser conocido fuera de Francia, viajando y dando Cursos y conferencias en la London School of Economics, en Inglaterra, lo mismo que en Alemania, Bélgica, Italia, o Noruega, entre otras partes. Sus artículos comienzan además a ser traducidos a otras lenguas, y a ser publicados en revistas o en libros de Bélgica, Escocia, Inglaterra o España, además de en múltiples revistas francesas. Y así, es esta primera difusión internacional de sus resultados de investigación la que lo lleva a impartir una serie de Conferencias, en 1929, en el Instituto para el Estudio Comparativo de las Civilizaciones de 0slo, conferencias que serán el origen de su importante libro titulado originalmente Les caracteres originaux de l'bistoire rural francaise. Un libro que verá la luz en 1931, y en el que Bloch va a reconstruirnos las etapas fundamentales y los elementos esenciales que conforman a la historia del mundo rural francés desde aproximadamente el año 1000 y hasta la víspera misma de la Revolución francesa.

Porque en esta obra mencionada no se trata solo de historia agraria o de la agricultura como actividad económica, sino mas bien de la caracterización social global de ese inmenso componente de la sociedad francesa que era su vasto universo constituido por su mundo rural, un universo rural que todavía en 1931 era sin duda dominante dentro del conjunto de dicha sociedad francesa. Entonces, y para lograr

${ }^{17}$ Sobre la historia específica de la escritura de esta fundamental obra de la historiografía del siglo XX, es posible ahora consultar la correspondencia de Marc Bloch con Henri Berr, Ecrire 'La Société féodale'. Lettres a Henri Berr 1924 - 1943, Ed. Institut Mémoires de l'édition contemporaine, Paris, 1992. 
establecer esa caracterización global, Marc Bloch revisa lo mismo las formas de ocupación del suelo y de transformación del paisaje y del territorio, que la condición de los grupos sociales y las clases fundamentales de ese mundo rural, pasando también por los trazos esenciales de la vida agraria, las formas de rotación de los cultivos, la figura de los campos o las formas de la propiedad agraria, y todo ello tanto en su conformación inicial como en sus respectivas curvas evolutivas a lo largo de prácticamente ocho siglos de historia.

Recuperando entonces lo mismo las lecciones de la geografía y de la economía, que de la sociología, la técnica, la toponimia, o la psicología, junto obviamente a la propia historia, Marc Bloch nos entrega en este libro sobre la Historia rural francesa todo un modelo de lo que debe ser una historia verdaderamente integral, crítica $y$ científica del universo rural de una sociedad cualquiera del planeta. Y si consideramos que el peso relativo de ese mundo rural es todavía, en la inmensa mayoría de la sociedades del mundo, de una magnitud considerable, comprenderemos el alcance y vigencia de esta clave histórico crítica contenida en este libro de Marc Bloch de $1931^{18}$. Una clave que, si en el caso de Bloch ha sido aplicada para descifrar la compleja historia de las muchas Francias que componen a Francia, y para permitirnos entender las razones principales de esa presencia aún masiva y omnipresente del mundo rural francés dentro de la dinámica global de la sociedad francesa de aquellos tiempos, en otros casos podría en cambio ser utilizada para explicar a la inmensa mayoría de las sociedades del llamado 'tercer mundo', en donde el peso enorme y hasta el predominio de ese componente rural sobre el conjunto social sigue siendo hasta hoy algo bastante frecuente.

También, vale la pena recordar que durante este periodo e incluso la mayor parte del siguiente, Marc Bloch va a tratar de superar la dramática experiencia de la primera guerra concentrándose en hacer bien y en desarrollar a fondo su propio trabajo de historiador. Es decir, de tratar de cumplir muy seriamente con sus tareas académicas y universitarias, y con sus proyectos intelectuales en general, aunque al precio de abstenerse en general de participar en la política, o de desarrollar mas a fondo sus deberes como ciudadano de una nación inmersa dentro de una Europa que atraviesa, como él mismo lo dirá después, una verdadera y profunda crisis de civilización. Y será el propio Marc Bloch el que, frente a la catástrofe del estallido de la segunda guerra, va a cuestionarse si ha hecho bien o no en abandonar ese frente de sus tareas ciudadanas que solo retomará a partir de 1939.

${ }^{18}$ Este libro ha sido traducido al español bajo el título Historia rural francesa. Barcelona: Grijalbo, 1978. El mismo ha servido de modelo, por ejemplo, para el libro de CHevaler, F. La formación de los grandes latifundios en México. México: FCE, 1975. 


\section{Los 'Primeros Annales' y la Revolucion en la Teoria DE LA HistORIA (1931 -1941)}

Desde 1929, el 15 de enero, había comenzado a aparecer regularmente en Estrasburgo una revista que, andando el tiempo, se convertirá en la mas importante revista de historia de Francia, de Europa, y de todo el entero mundo occidental. Una revista fundada por Marc Bloch y por Lucien Febvre, titulada inicialmente Annales d'Histoire Economique et Sociale, que en primer lugar, va a instaurar dentro de Francia, con pleno derecho de ciudadanía y con toda vigencia, a las nuevas ramas de la historia económica y de la historia social, ya bastante desarrolladas en otros países de Europa para esas fechas, pero que en Francia se habían retrasado en cuanto a su afirmación y desarrollo mas generales. Con lo cual, esos Annales de la primera época, codirigidos por Bloch y por Febvre, no solo servirán de verdadero laboratorio para la instauración realmente orgánica de estas ramas de la historia económica y social mencionadas, sino también y mas generalmente, como un polo concentrador y difusor de esa misma contribución francesa a este campo reciente de la historiografía europea y mundial.

En segundo lugar será gracias a este proyecto de los Annales y a través de él, que va a realizarse justamente el desplazamiento del epicentro de los estudios históricos de aquella época, desde su antiguo emplazamiento dentro del espacio cultural germanoparlante, hasta ese nuevo territorio cultural que seráel propio hexágono francés. Porque luego del derrumbe de la cultura y de la historiografía alemanas, provocado por la primera guerra mundial y sobre todo por el ascenso de Hitler al poder, va a comenzar a gestarse una nueva begemonía cultural y también bistoriográfica que, entre 1929 y 1968 aproximadamente se vinculará de manera directa con la propia cultura e historiografía francesas. Y dentro de la historiografía, el vehículo central de esta nueva hegemonía será sin duda el proyecto intelectual de estos mismos Annales.

En tercer lugar, estos Annales de Bloch y Febvre van a llevar a cabo una verdadera crítica radical y un desmontaje teórico de la historia positivista, entonces dominante en La Sorbonne y en todo el ámbito historiográfico francés, a través de la realización de una auténtica revolución en la teoría de la bistoria, que abarcará lo mismo una nueva definición del objeto global de estudio de la historia y una nueva concepción del sentido mismo de lo que implica el ejercicio de esta, que el establecimiento de todo un conjunto de nuevos paradigmas metodológicos, de nuevos modelos, teorías y conceptos históricos, de nuevos temas y campos problemáticos de investigación y de nuevas explicaciones 0 interpretaciones de los viejos y de los nuevos temas historiográficos abordados. 
Revolución en la teoría de la historia que desplegándose en todos esos frentes teóricos, metodológicos, problemáticos e historiográficos va a constituir el núcleo central del excepcional proyecto crítico de los primeros Annales, que abarcando toda la década de los años treintas, solo se cerrarácon la dura disputa y ruptura de la primavera de 1941, en la que Marc Bloch y Lucien Febvre se opondrán diametralmente respecto de la decisión de, o interrumpir la publicación de los Annales parasalvaguardar su independencia ideológica y cultural en general, que ha sido la posición defendida por Bloch, o continuar su publicación sometiéndose a las condiciones dictadas por la censura nazi entonces instalada en Paris, que ha sido la postura de Febvre y que serála que finalmente prevalecerá ${ }^{19}$. Y es claro que el libro blochiano de la Apología para la historia o el Oficio de Historiador, va a constituir también, entre otras varias de sus dimensiones esenciales, una verdaderay muy lograda sintesis metodológica de las principales lecciones y de los principales aportes contenidos en esos 'primeros' Annales de 1929-1941, codirigidos realmente por el propio Bloch junto a Lucien Febvre.

Impulsando entonces desde esos primeros Annales los nuevos paradigmas de una historia interpretativa, comparatista, global, y concebida como 'historia-problema' y como historia en construcción, Marc Bloch va a ir desarrollando lo que serála dimensión metodológica esencial de ese proyecto fundador de la corriente francesa que, andando el tiempo, terminará por ser la mas importante corriente de la historiografía francesa en todo el siglo veinte. Dimensión metodológica que, no casualmente, va a defender dentro de Francia y dentro de una parte importante de Europa, toda una serie de postulados y de principios de método que, al ser observados con cuidado, presentan una gran similitud con varias de las tesis centrales y de los paradigmas metodológicos del proyecto crítico del marxismo también fundador y original20.

Simultáneamente a este trabajo vasto y continuado de verdadera construcción de los Annales, Marc Bloch va a ir acrecentando la red internacional de sus interlocutores

19 Sobre el significado profundo y sobre las premisas esenciales de esta radical ruptura de 1941 cf. nuestro libro Aguirre Rojas, C.A. La Escuela de los Annales. Ayer; Hoy, Mañana, en especial el capítulo 3, pp. 93-104, antes citado. También en este libro hemos desarrollado con mucha mas amplitud los contenidos de la referida 'revolución teórica' de esos primeros Annales, que aquí solo enunciamos brevemente.

${ }^{20}$ Sobre estas similitudes entre los paradigmas de los primeros Annales y el marxismo cfr. nuestros libros antes citados, AguirRe RojAs, C.A. Los Annales y la bistoriografía francesa, y también Antimanual del mal bistoriador. Y esta cercanía intelectual entre ambos proyectos será tan clara, que incluso después de 1968 ha dado origen a un doble movimiento de mutuo acercamiento, con marxistas que recuperan el legado de los Annales del periodo 1929 - 1968, y con analistas que se acercan fructíferamente al marxismo, conformando en ocasiones los elementos de una posición o matriz historiográfica que bien podríamos calificar de 'marxistaannalista' o de 'annalista-marxista', como hemos ya postulado en nuestro libro La Escuela de los Annales. Ayer; Hoy, Mañana. 
y de los colegas dedicados a los mismos temas que él investiga con los que establecerá contactos regulares, enriqueciendo de este modo los apoyos e insumos diversos de sus distintas investigaciones. Contactos intelectuales y diálogo cultural que se multiplicarán y agilizarán a partir de 1936, cuando Marc Bloch consiga finalmente ganar la cátedra de historia económica en La Sorbonne que ha dejado vacía Henri Hauser, trasladando por esta vía toda su actividad y todas sus labores de investigación a la ciudad de Paris. Labores de investigación que, en estos años parisinos previos al estallido de la segunda guerra, irán confluyendo cada vez más hacia la redacción definitiva de los dos volúmenes de su importante libro La Sociedad Feudal.

Este libro, cuyo primer volumen será publicado en 1939, y cuyo segundo volumen verá la luz en 1940, en plena segunda guerra, es un libro que sin duda debería figurar en la lista de los diez mas importantes libros de historia escritos durante el siglo XX. Un texto que será sin duda la obra histórica mas importante salida de la pluma de Marc Bloch, y que es en verdad la presentación de un modelo global de explicación de la estructura social del mundo y de la civilización europeos durante su especifica etapa medieval. Un modelo de interpretación de esta etapa infantil o formativa de la civilización europea, que ha sido el periodo feudal de su historia, que no ha sido aún superado, sesenta años después, por ninguno de los ulteriores estudiosos franceses, europeos o de cualquier otra parte del mundo, de esta misma historia medieval.

Porque mas allá de los cientos de monografías y de las decenas de libros que en estas últimas seis décadas se han dedicado a investigar tal o cual aspecto particular de dicha sociedad feudal, subsiste el hecho de que ningún historiador o científico social ha vuelto a intentar proponer otra síntesis global comparable a ese texto de La Sociedad Feudal, que abordando el mundo feudal como totalidad, se atreviese a abarcar nuevamente todas las diversas dimensiones que Bloch ha investigado tan magistralmente en su obra, conectándolas e integrándolas de manera coherente en un solo y universal modelo global e integral de interpretación.

Ya que como lo ha dicho el mismo Marc Bloch, lo que en este trabajo se encuentra, es en verdad el modelo de análisis de una estructura social cualquiera, que aquí se ha aplicado a dicha sociedad feudal, pero que en el futuro podría también aplicarse a otras sociedades, o también a otros periodos de la misma historia de la civilización europea. Un modelo que comprende, estrictamente hablando, todas las dimensiones de dicha estructura social, desde su configuración geográfica espacial y su inscripción dentro del territorio, y el establecimiento de su modo de apropiarse a la naturaleza en términos productivos y tecnológicos, hasta sus cosmovisiones culturales, sus creencias colectivas 
diversas, o sus formas de hacer la guerra, pasando por los distintos elementos componentes de su organización económica, su estructura de clases sociales determinada, o sus formas de configuración y de articulación del poder político, pero también y junto a todo esto, por sus estructuras de parentesco, las formas de sus linajes y los modos de funcionamiento de la familia, por la forma de sus leyes y normas jurídicas, por las distintas dimensiones y expresiones de su vida cotidiana, o de sus productos literarios, artísticos o religiosos, lo mismo que su vocabulario, la nomenclatura de sus ciudades, sus formas originarias de poblamiento, sus costumbres, su memoria, su atmósfera mental, sus formas de sentir y de pensar, o su específica manera de percibir el tiempo, entre tantos otros de los temas y de las dimensiones abordados en este complejo y elaborado fresco globalizante sobre la sociedad feudal medieval europea occidental ${ }^{21}$.

Verdadero modelo de lo que debe ser una historia global, este libro de La Sociedad Feudal de Marc Bloch ha logrado, como uno de sus efectos intelectuales principales, terminar con la visión romántica todavía prevaleciente en la mayoría de los ambientes académicos de la Europa de principios del siglo XX, que consideraba al feudalismo solo como una 'edad obscura' y como una clara etapa de retroceso social general, como una 'Edad Media o Intermedia' entre la luminosa Antigüedad clásica y el brillante Renacimiento europeo, y por lo tanto como muy poco digna de estudio o de examen histórico mas en especial. Así, inscribiéndose en la misma línea de los efectos producidos por la obra de Henri Pirenne y de Alphons Dospsch, esta obra de Bloch ha terminado de relegitimar el periodo de la historia medieval europea, el que desde sus investigaciones comenzará a ser mucho mas frecuentado e investigado en Francia y en toda Europa.

Igualmente, y junto a esa condición ejemplar como estudio de historia global, este libro blochiano es también una aplicación paradigmática del método comparativo en la bistoria, que nos solo construye para nosotros el concepto general de 'mundo o sociedad feudal' desde la minuciosa y paciente comparación de los diversos feudalismos francés, alemán, inglés e italiano, sino que también es capaz de darnos, sucesivamente y de una manera rica y sutil, la explicación específica de lasparticularidades que presentan y que explican en cada caso los casos de ese mundo feudal en los diversos espacios de lo que mas adelante serán España, Inglaterra, Francia, Alemania o Italia.

De este modo, y cuando Bloch está todavía revisando las últimas pruebas de este libro sobre La Sociedad Feudal, estalla la segunda guerra mundial, que no solo provocará la ruptura entre Bloch y Febvre en 1941, y con ella el fin del rico proyecto intelectual de

${ }^{21}$ Cf. Bloch, M. La Sociedad Feudal. México: Uteha, , 1979. 2 vols. También Aguirre Rojas, C.A. "Feudalismus" en el libro Historisch-Kritisches Wöerterbuch des Marxismus. Berlin: Argument, 1999. 
los primeros Annales, sino también un cambio radical y dramático de la situación general del mismo Bloch, quien a pesar de estar doblemente exento de ser movilizado en dicha segunda guerra, por su edad y por ser el padre de seis hijos, decidirá de una manera totalmente libre y voluntaria participar en ella, haciendo de lado todas sus actividades académicas e intelectuales, y sacrificando todas sus comodidades y su situación en virtud de una clara conciencia de su deber como ciudadano y como francés.

\section{Los Ultimos AÑos (1941 - 1944)}

Cuando en 1939, Marc Bloch decide participar en la segunda guerra, no puede adivinar que sus habilidades y sus conocimientos serán subutilizados cuando le sean asignadas tareas demasiado simples y rutinarias, durante todo el primer año de la guerra y hasta el armisticio de 1940. Y sin embargo, esa actividad demasiado elemental y burocrática que le ha sido encomendada, quizá le haya permitido gozar del suficiente tiempo libre como para comenzar a reflexionar, críticamente, en torno de las razones generales que explican la tan rápida derrota francesa frente al enemigo alemán ${ }^{22}$.

Porque durante el verano de 1940, y luego del mencionado Armisticio, Bloch se dará a la tarea de redactar ese Testimonio escrito en 1940, que seis años mas tarde y luego de terminada la segunda guerra mundial, serápublicado en 1946 por las Ediciones Atlas, creadas por el movimiento francés 'Franc-Tireur', bajo el título ahora muy conocido de La Extraña derrota.

Porque es claro que a partir de esta derrota francesa, Bloch a comenzado a interrogarse directamente respecto del papel que él mismo, y mas general todo el sector de los intelectuales franceses, han jugado durante los años de entre las dos guerras mundiales que corren desde 1919 hasta 1939. Y su respuesta clara es que su generación es una generación que tiene 'mala conciencia', porque luego de haber vivido y tomado partido dentro del Affaire Dreyfus, y de haber participado en la primera guerra mundial, ha vuelto en 1919 "demasiado fatigada" como para continuar participando en los asuntos políticos, olvidándose de sus deberes ciudadanos y dejando hacer y asumir libremente a los gobernantes y a los políticos las tareas y responsabilidades que también le correspondían a ella misma.

${ }^{22}$ La situación que ha vivido Marc Bloch durante este primer año de la guerra, antes del Armisticio y la derrota francesa, se encuentran reflejadas en las cartas que le ha escrito a su hijo Etienne, y que están publicadas en francés bajo el título Marc Bloch a Etienne Bloch. Lettres de la 'drole de guerre', Cahier 19 de la serie Les Cahiers de l'IHTP. Paris: Institut d'Histoire du Temps Present, decembre, 1991. 
Con lo cual, también estos intelectuales franceses, - que aunque disponían de 'una lengua, una pluma, un cerebro' para influir en la opinión pública y para fijar claramente su posición, no lo ban becho -, son en parte responsables de esa misma derrota francesa. Pues si el intelectual no asume su compromiso social con el propio presente y con la sociedad en los que vive, se hace igualmente responsable, por omisión, del destino y de los rumbos que tome esa misma sociedad en el momento de ir hacia el encuentro de su particular futuro.

Acuciado entonces por esta preocupación sobre el compromiso ciudadano y social del intelectual, Bloch redacta en 1940 ese libro sobre La Extraña derrota, en el que la pregunta central a responder es justamente la de ¿por qué Francia ha sido derrotada?. Y para responderla, nuestro autor va a realizar un diagnóstico crítico del conjunto de la sociedad francesa en vísperas de la segunda guerra, que además de agudo e implacable, va a resultar todavía aleccionador respecto de lo que ha sido la historia de la nación francesa durante todo el siglo XX recién concluido. Porque en su análisis Bloch va a caracterizar con mucha agudeza lo mismo al Estado Mayor militar francés, y a las clases dirigentes de Francia, que a los sindicatos, los partidos políticos, las Universidades y a los propios ciudadanos del hexágono. Yello, lo mismo para mostrar el increíble retraso de la elite militar, que se quedó anclada en las lecciones de la primera guerra sin actualizarse en torno a los nuevos desarrollos de la estrategia militar, que los vicios de la burocracia francesa, siempre enorme y siempre ineficiente, junto a las lagunas y limitaciones del sistema escolar y del tipo de formación impulsado en las escuelas, la cortedad de miras de los partidos políticos o de los sindicatos, o las consecuencias negativas de la inercia y de los límites de muchas tradiciones albergadas y conservadas dentro de las 'pequeñas aldeas' de una cierta Francia campesina y profunda ${ }^{23}$.

Después de redactar este testimonio lúcido y preocupado, Marc Bloch va a comenzar a vivir toda una serie de cambios y catástrofes personales que, al mismo tiempo que radicalizan poco a poco su conciencia política, van a determinar también la naturaleza de sus últimos trabajos intelectuales y de sus últimas actividades en general. Pues después de este verano de 1940 Bloch será víctima del Estatuto contra los Judíos que lo obligará a abandonar su Cátedra en la Sorbonna y la dirección del Instituto de Historia Económica y Social, y con ellos, mas adelante, la propia ciudad de Paris. Vendrá luego la ruptura con

${ }^{23}$ Es curioso mencionar que este libro de La extraña derrota fue muy poco conocido y leído hasta antes de 1990. Pues a pesar de haber sido publicado en 1946 por las Ediciones Atlas, y reeditado en 1957 por la Armand Colin, su circulación se mantuvo muy restringida. Solo después de la reedición de 1990 por parte de la Gallimard, en una colección de bolsillo, fue que comenzó a ser mas discutido e incorporado en los análisis sobre la obra de Marc Bloch. Como ya hemos señalado, aún no existe una traducción al español de este libro. 
Lucien Febvre, en la primavera de 1941, ruptura que implicará que Bloch no vuehia nunca más a colaborar en los Annales con la misma intensidad y con el mismo compromiso que antes de esta misma fecha. También resultará fallida la tentativa de emigrar con toda su familia a Estados Unidos ${ }^{24}$, y en mayo de 1942, será allanado su departamento parisino por la policía nazi, lo que conllevará la pérdida de una gran parte de su Biblioteca y de sus fichas, sus notas de lectura, sus dossier y materiales habituales de trabajo. Finalmente, y luego de verse obligado por diferentes razones a moverse hacia Clermont-Ferrand y a Montpellier, para proseguir allí con su actividad docente, terminará abandonando esta última después de la ocupación total de Francia por lo alemanes, refugiándose primero en su casa de campo de Fougeres, y trasladándose finalmente a Lyon, para desarrollar allí actividades políticas de tiempo completo dentro de los Movimientos Unidos de la Resistencia francesa contra los nazis.

Y es justo en este contexto de tales cambios radicales de situación personal, y dentro de las condiciones-límite que representa la segunda guerra mundial, que Marc Bloch va a acometer, entre 1941 y 1943, la redacción de su inconclusa obra Apologie pour l'Histoire ou Métier d'Historien. Una obra que entonces no es el fruto de una escritura realizada en condiciones serenas, tranquilas y apacibles, sino mas bien el esfuerzo intelectual apremiante de responderse y responder a la pregunta de 'ipara qué sirve la historia?' en las difíciles circunstancias de una civilización que se viene abajo y se autoinmola masivamente entre 1939 y 1945 . Es decir, el intento complicado pero necesario de preguntar qué sentido puede tener la profesión de historiador, y que elementos útiles puede aportar esa misma práctica histórica a una sociedad que se desmorona frente a nuestros ojos, enmedio de la brutal crisis de una guerra sin precedentes.

Entonces, y desde este horizonte particular, es que Bloch va a condensar en ese bello e incompleto libro de la Apología por la Historia, sus reflexiones de décadas en torno al objeto de la ciencia histórica, a los métodos y técnicas de esta misma ciencia, a la definición de lo que es un hecho histórico y lo que implica su examen e interpretación adecuada por parte del historiador, al enorme problema del tiempo histórico y de las relaciones entre el pasado y el presente, a los elementos y exigencias del método crítico aplicado a los testimonios y fuentes historiográficas, lo mismo que al papel de la mentira y del error en la historia, a la naturaleza de las verdades históricas, a la fragilidad de los

\footnotetext{
${ }^{24}$ Están publicadas las cartas de Marc Bloch a sus corresponsales americanos, en torno de este fallido proyecto de emigrar a los EUA, para ir a trabajar a la New School for Social Reserach en Nueva York. Cf. RuTkoff, P. y ScotT, W. Letters to America: The correspondence of Marc Bloch, 1940 1941, en la revista French Historical Studies, 12, 1981.
} 
testimonios, a las virtudes y requerimientos del método comparativo, o al papel esencial del 'cuestionario' en historia, entre tantos y tantos otros puntos allí abordados.

Porque como ya hemos señalado, ese texto sobre el Oficio de Historiador, no es solo la síntesis de todo el rico y variado itinerario intelectual de Marc Bloch que aquí hemos esbozado, sino también la clara condensación de la rica experiencia de los primeros Annales, aquí decantada en lo que corresponde a sus principales lecciones metodológicas. Pero también y en un plano mas profundo, esta última obra de Marc Bloch va a convertirse igualmente en el resumen de los progresos que la ciencia histórica francesa y europea conquistó durante la primera mitad del siglo XX, y que en virtud del cambio de hegemonía historiográfica que ya hemos señalado antes, confluyeron también en gran medida dentro de esos iniciales Anales d'Histoire Economique et Sociale anteriores al año de $1941^{25}$.

Un libro extraordinario de metodología histórica, con lecciones que siguen siendo profundamente actuales y vigentes, que quedará inconcluso debido a la aguda conciencia de Bloch de lo que implica el compromiso social y político de un intelectual serio, honesto y realmente científico. Porque cuando los nazis invaden la zona todavía libre de Francia, y cuando la amenaza que ellos representan se vuelve mas clara y evidente, entonces Marc Bloch decide abandonar sus reflexiones sobre la historia para dedicarse de tiempo completo al trabajo de la resistencia antinazi. Y entonces, ese hombre excepcionalmente inteligente, que es sin duda el mas importante historiador occidental de la primera mitad del siglo XX, se consagra al trabajo político clandestino en la ciudad de Lyon, siendo aprendido el 8 de marzo de 1944, salvajemente torturado, y finalmente asesinado el 16 de junio en las orillas del pequeño pueblo de Saint-Didier-des Formans. Cerrando de este modo, con esa muerte profundamente absurda e injusta su propio itinerario, Marc Bloch nos ha legado sin embargo, tanto una obra que sigue siendo imprescindible para los verdaderos historiadores críticos, como también un ejemplo digno de reflexión para todos aquellos intelectuales realmente honestos, serios e igualmente críticos, que no consideran a la ciencia como un simple pasatiempo, sino mas bien como una actividad digna, humana y realmente social.

\footnotetext{
${ }^{25}$ Ya hemos mencionado antes la reciente edición de una mueva versión, mas completa y mas rica, de esta Apología para la Historia u Oficio de Historiador, editado en México por el Fondo de Cultura Económica en 1996. Sobre las distintas dimensiones que comprende este libro y sobre su excepcional difusión en América Latina, cf. nuestro ensayo "La recepción del Metier d'Historien de Marc Bloch en América Latina". Argumentos, 26. México, antes citada.
} 